\title{
Real-Time Data Mining Models for Predicting Length of Stay in Intensive Care Units
}

\author{
Rui Veloso ${ }^{1}$, Filipe Portela ${ }^{1}$, Manuel Filipe Santos ${ }^{1}$, Álvaro Silva², Fernando Rua ${ }^{2}$, António Abelha ${ }^{3}$, \\ José Machado ${ }^{3}$

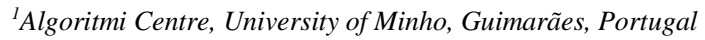 \\ ${ }^{2}$ Serviço Cuidados Intensivos, Centro Hospitalar do Porto, Hospital Santo António, Porto, Portugal \\ ${ }^{3}$ CCTC, University of Minho, Braga, Portugal \\ ruiveloso51046@gmail.com, \{cfp,mfs\}@dsi.uminho.pt, moreirasilva@gmail.com, \\ fernandorua.sci@hgsa.min-saude.pt, \{abelha,machado\}@di.uminho.pt
}

Keywords: $\quad$ Length of Stay, INTCare, Intensive Care Units, Data Mining, Real-Time

Abstract: Nowadays the efficiency of costs and resources planning in hospitals embody a critical role in the management of these units. Length Of Stay (LOS) is a good metric when the goal is to decrease costs and to optimize resources. In Intensive Care Units (ICU) optimization assumes even a greater importance derived from the high costs associated to inpatients. This study presents two data mining approaches to predict LOS in an ICU. The first approach considered the admission variables and some other physiologic variables collected during the first 24 hours of inpatient. The second approach considered admission data and supplementary clinical data of the patient (vital signs and laboratory results) collected in real-time. The results achieved in the first approach are very poor (accuracy of $73 \%$ ). However, when the prediction is made using the data collected in real-time, the results are very interesting (sensitivity of 96.104\%). The models induced in second experiment are sensitive to the patient clinical situation and can predict LOS according to the monitored variables. Models for predicting LOS at admission are not suited to the ICU particularities. Alternatively, they should be induced in real-time, using online-learning and considering the most recent patient condition when the model is induced.

\section{INTRODUCTION}

With a constant increase in health expenses and the aggravation of the global economic situation, managing costs and resources in healthcare assumes an important role. In Intensive Care Units (ICU) this reality is even more felt because of the costs associated to each patient. These costs are quite high due to several factors as is the large number of equipment used, the high number of nurses and doctors, the great variety of therapies and drugs administered and the patient Length of Stay (LOS). All these factors lead to an increase of daily patient costs in an ICU. Planning and provisioning resources and costs in ICU assumes a critical role in hospital management (Marshall et al., 2005). Accurate predictions of the patient's LOS in the ICU can contribute to reduce costs. It is recognized by the ICU professionals the importance of having a tool which help to predict the patient's LOS. This can help managing the resources and better understand the patient condition, contributing to avoid wrong discharges.

Related works found in literature are not accurate for ICU setting, due to patient specific conditions. Consequently, the principal motivation of this research is to induce data mining models that can predict accurately the LOS of ICU inpatients.

The idea of this work is to understand how many days a patient with some clinical diseases need to be in the unit. This only it is possible having a system which can in real-time adapt the patient's LOS according to the patient condition. With a correct prediction of LOS it is possible make a better resources allocation and consequently decrease the patient costs, for example, the unit will have a better professional's allocation and the therapeutics (dosages and schedules) can be prescribed according to the patient LOS. To reach the objectives, two approaches were explored using Data Mining (DM) in order to predict the probability of a patient be discharged in the next hours. The first approach (A) considered data acquired in the first twenty-four 
hours and the second approach (B) used data acquired in real-time. Although the two approaches have the same goal it is very difficult to compare them, because the way how the goal was achieved differs. The approach A predicted a LOS class and the Approach B predicted the probability of a patient be discharged in the next hour. The second approach was explored only because approach A failed. For a better understanding of the work, the two approaches will be evaluated separately.

Approach A used data from the patient admission plus some clinical variables and presented weak results. These models used classification techniques to predict the best LOS class. The overall accuracy of these models ranged from $7.60 \%$ to $73.28 \%$. For approach A is not possible to determine sensitivity and specificity because involves more than two output classes. Sensitivity has a great importance in clinical decision because physicians look for models sensible to one result. The models, which predict patient discharge hourly, revealed good results. Mostly all of the models presented accuracies upper than $70 \%$ and in some cases sensitivities upper than $90 \%$. This work was based in the study started by INTCare project.

This article is divided into five sections. The first one introduces the paper. The main concepts and related work are described in the background in the section two. In the third one is described the study, data analysis, transformation, modelling and results. The subsequent section discusses the results obtained. The last section makes final considerations and proposes future work.

\section{BACKGROUND}

\subsection{Length of Stay}

Expenditures in hospital care are rising and the costs associated to the patient represent a significant portion of the expenses. Reducing Length of Stay (LOS) in hospitals will lead to an effective reduction in the costs and an effectiveness improvement in hospitals (Clarke, 1996). The LOS is the number of days that a patient is subjected to treatment since their admission date until the discharge date. This number of days is counted if the patient is at home or at the hospital, depending where he is receiving health cares. The use of this indicator permits to see the costs with the patient or group of patients along as the quality of the services given to the patients (Marshall et al., 2005). Patients with outsized LOS in the ICU represent an enormous usage of resources so a reliable prediction of LOS can lead to a rational bed allocation and to a resources maximization (Wagener et al., 2011), being more efficiently.

Several works can be found about LOS but for predicting LOS in ICU no one was found. Isken and Rajagopalan (2002) use clustering techniques and kmeans algorithms to identify types of patients so that can be constructed computational simulations or analytic models about patient flow inside hospitals. These flow models consider the analysis of measures like LOS and bed occupancy rate. Another work used classification techniques recurring to decision three algorithms, support vector machines and artificial neural networks to predict LOS in patients with cardiac disease (Hachesu et al., 2013). Azari (Azari et al. 2012) propose a multi-layer approach to predict LOS in hospitals. The work used clustering techniques to build the training data set. Then, several classification algorithms were used with the Naïve Bays, SVM, JRIP and J48 showing the best results. In the area of Gastroenterology (Zhang et al. 2012) were developed models to predict LOS in ICU in post-operatory elderly patients with gastric cancer. The study used regression trees to predict LOS and other hospital metrics. Caetano et al. (2014) proposed a data-driven predictive model to LOS with data from Portuguese hospitals from 2000 to 2013 and used as inputs the typical available indicators at the hospitalization process. The work developed several DM Models with the best results being achieved with Random Forest methods.

\subsection{INTCare}

This study is being developed under the research project INTCare. INTCare is a Pervasive Intelligent Decision Support System (PIDSS) that is in constant testing and development. Actually it is implemented in the ICU of the Hospital de Santo António, Centro Hospitalar Porto. This PIDSS is based on intelligent agents (Santos et al., 2011) and aims to support the decision making process and predict clinical events anywhere and anytime like patient organ failure (cardiovascular, respiratory, renal, hepatic, neurological and hematologic), patient outcome (Portela et. al, 2013a), readmissions, medical diseases and others. Regarding the predictions made the system is able to support the decision on procedures, treatments and therapies. Predictions are made by means of DM techniques. The system is based in four autonomous subsystems (data acquisition, knowledge management, inference and interface) that use intelligent agents to perform their actions (Portela et al. 2013b, Portela et al., 2012). 


\subsection{Data Mining}

Data Mining (DM) is a field that uses techniques and methods in data to find trends or patterns in order to support the discovery of new knowledge (Santos \& Azevedo, 2005). DM identifies opportunities or threats in business, to support decision making process or to solve problems. Among the best-known application areas are Customer Relationship Management (CRM), banking, retail and logistics businesses, the entertainment industry, sports, insurance, travel industry (airlines, hotels, car rental), health and medicine (Turban, 2010). A classification model can be viewed as a function for mapping new cases (input) to a class from a list of classes (output) (Santos \& Azevedo, 2005). For example, these type of models can be used to classify the banking history of users as good or bad (Rokach \& Maimon, 2010).

\subsubsection{Decision Trees}

Decision Trees (DT) was introduced in 1986 in the machine learning area with the use of ID3 algorithm to synthesizing decision trees (Quinland, 1986). A DT based classifier is a partition of an instance of a space. The decision trees are composed by nodes and branches. Usually, the DT is constructed with a top-down strategy with the first node, the root node, don't having incoming nodes and giving origin to two or mode nodes according to the attributes that are being used. The remaining nodes have only one incoming node. The nodes that give origin to others are called internal or test nodes and the others are called leaves or terminal nodes (Hand, 2001). Figure 1 represents the concept of DT.

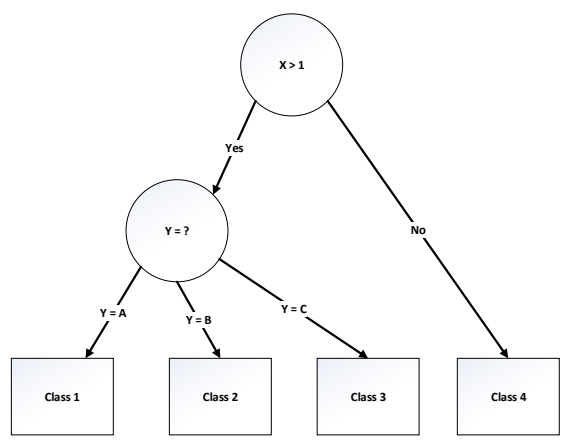

Figure 1: Decision Tree, adapted from (Kantardzic, 2011).

The models developed using DT technique produced rules in the form of IF predictive information THEN target (Taylor, 2010). These models use for example CART algorithm that is a DT learning technique to produce DT as a binary recursive partitioning procedure capable of processing continuous and nominal attributes as targets and predictors (Breiman et al, 1984).

\subsubsection{Naïve Bayes}

One of the first implementations of a Naïve Bays classifier occurred in 1994 (Michei et al, 1994).

Bayesian classification is a technique to the classification of patterns in a set of data. This type of technique assumes that the classification of patterns is expressed in probabilistic terms. The classification based on Bayes theory pretend to classify objects based on the statistical information of the objects to minimize the probability of the classification is done poorly (Cios et al. 2007). The Bayes theorem can be expressed by the following expression:

$$
\mathrm{P}(\mathrm{Ci} \mid \mathrm{X})=(\mathrm{P}(\mathrm{X} \mid \mathrm{Ci}) * \mathrm{P}(\mathrm{Ci})) / \mathrm{P}(\mathrm{X})
$$

Where,

$\mathrm{P}(\mathrm{Ci} \mid \mathrm{X})$ represents the a posteriori probability;

$\mathrm{P}(\mathrm{Ci})$ represents the a priori probability;

$\mathrm{P}(\mathrm{X} \mid \mathrm{Ci})$ probability density function $\left(\mathrm{C}_{\mathrm{i}}\right)$;

$\mathrm{P}(\mathrm{X})$ unconditional probability density function.

\subsubsection{Support Vector Machines}

Support Vector Machines (SVM) are classification methods to map vectors into a space using nonlinear mapping building an optimal separating hyperplane (Vapnik, 1995). The optimal hyperplane is obtained when the hyperplane is as far as possible from all the data points that compose the space. SVM algorithms extend the measurement space in more complex surfaces so the space includes combinations of the raw variables. Then trough the margin, the algorithm attempt to optimize the location of the linear decision boundary between two classes that is likely to lead to the best possible generalization, using the biggest margin (Hand et al, 2001). Figure 2 represents a set of data that can be linearly separable and where exists an infinite number of hyperplanes and decision boundaries.

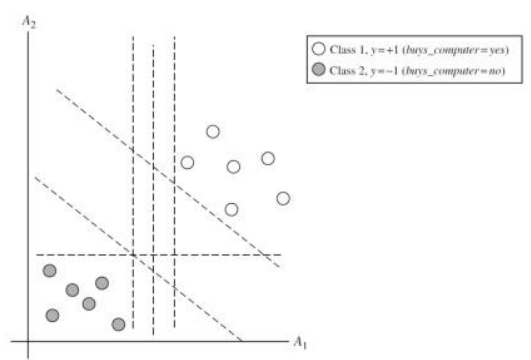

Figure 2: Hyperplanes and Decision Boundaries, adapted from (Han, 2011) 


\subsubsection{CRISP-DM}

Once this study is a data mining work it was followed the CRISP-DM methodology. CRISP-DM is an acronym for Cross Industry Standard Process for Data Mining. This paper is organized around the first five tasks of this methodology: business understanding, data understanding, data preparation, modelling and evaluation (Chapman et al., 2010).

\section{STUDY DESCRIPTION}

\subsection{Business Understanding}

As mentioned above, the prediction of LOS assumes an important role on the optimization of costs and resources in the ICU. In these units a lot of attributes are available to be used for predicting LOS. Two approaches were experimented in this study for predicting LOS of patients in the ICU. The first approach (A) uses as input data values of collected in the first 24 hours considering data like the admission type (worst vital signs values). This approach is transversal to many areas in medicine but is a bad choice when used to predict LOS in intensive medicine. Alternatively, a second approach (B) was explored. This approach considers more variables and intends to predict the LOS based in the probability of a patient be discharged in the next hour. Then, the LOS is obtained by finding the hour with the higher probability of the patient be discharge. This hour represents the inpatient time.

Both approaches used Oracle Data Mining (ODM) and considered three of the classification DM techniques available: Decision Trees, Support Vector Machines and Naïve Bayes.

\subsection{Data Understanding and Transformation}

\subsubsection{Approach A}

The acquisition of the data used in this approach is made from the electronic health record, laboratory, and vital signs monitor. Table 1 presents some statistics about the variables considered.

Table 1: Variables used in approach A.

\begin{tabular}{|r|l|r|r|r|}
\hline \multicolumn{1}{|c|}{ Variable } & \multicolumn{1}{|c|}{ Min } & Max & Value \\
\hline ID & Age & 20 & 96 & Real \\
\hline Sex & Sex & M & F & 1 or 2 \\
\hline Admission Type & Urgent & - & - & $\mathrm{U}$ \\
\hline
\end{tabular}

\begin{tabular}{|c|c|c|c|c|}
\hline & Programmed & - & - & $\mathrm{P}$ \\
\hline \multirow{2}{*}{ Admission surgery } & With & - & - & $\mathrm{W}$ \\
\hline & Without & - & - & $\mathrm{WO}$ \\
\hline \multirow{7}{*}{ Admission From } & Chirurgic & - & - & 1 \\
\hline & Observation & - & - & 2 \\
\hline & Emergency & - & - & 3 \\
\hline & \begin{tabular}{|l|} 
Nursing Room \\
\end{tabular} & - & - & 4 \\
\hline & Other ICU & - & - & 5 \\
\hline & Other Hospital & - & - & 6 \\
\hline & Other & - & - & 7 \\
\hline Glasgow Coma & GCS & 3 & 15 & Real \\
\hline \multirow{2}{*}{$\begin{array}{r}\text { Hematologic } \\
\text { Disease } \\
\end{array}$} & Yes & - & - & $\mathrm{Y}$ \\
\hline & No & - & - & $\mathrm{N}$ \\
\hline \multirow{2}{*}{ Transplanted } & Yes & - & - & $\mathrm{Y}$ \\
\hline & No & - & - & $\mathrm{N}$ \\
\hline \multirow{2}{*}{ HTA in Treatment } & Yes & - & - & $\mathrm{Y}$ \\
\hline & No & - & - & $\mathrm{N}$ \\
\hline \multirow{2}{*}{$\begin{array}{r}\text { Insulin Treated } \\
\text { Diabetes } \\
\end{array}$} & Yes & - & - & $\mathrm{Y}$ \\
\hline & No & - & - & $\mathrm{N}$ \\
\hline Non-Insulin Treated & Yes & - & - & $\mathrm{Y}$ \\
\hline Diabetes & No & - & - & $\mathrm{N}$ \\
\hline Days of Inpatient & Inpatient Days & 0 & 40 & Real \\
\hline Hours of Inpatient & Inpatient Hours & 2 & 970 & Real \\
\hline Oxygen Saturation & SPO2 & 86.00 & 100.00 & Float \\
\hline $\begin{array}{r}\text { Systolic Blood } \\
\text { Pressure } \\
\end{array}$ & SYS & 80.64 & 219.48 & Float \\
\hline $\begin{array}{r}\text { Diastolic Blood } \\
\text { Pressure } \\
\end{array}$ & DIA & 43.20 & 179.88 & Float \\
\hline $\begin{array}{r}\text { Corporal } \\
\text { Temperature } \\
\end{array}$ & TEMP & 34.16 & 39.74 & Float \\
\hline Heart Rate Measure & EGG_HR & 50.00 & 231.00 & Float \\
\hline
\end{tabular}

Case Mix consists in a set of variables that are present in the Electronic Health Record of the patient. In this approach were included the variables age, admission type and admission from, admission with surgery, Glasgow coma scale, hematologic disease, transplanted, HTA in treatment, insulin treated diabetes, non-insulin treated diabetes, hours and inpatient days. These variables are obtained in the moment of admission. Other variables were considered (physiological variables) and collected during the first 24 hours. From the vital signs monitor, the following variables were collected: Oxygen Saturation (SPO2), Systolic Blood Pressure (SYS), Diastolic Blood Pressure (DIA), Corporal Temperature (TEMP) and Heart Rate Measure (EGG_HR). In this approach, they were introduced some classes for the variables days and inpatient hours (2 classes for each variable), EGG_HR, SPO2, ART_SYS and ART_DIA by dividing the cases of each class by equal number. The variables age, sex, admission type, admission with surgery, admission from, Glasgow coma scale, hematologic disease, transplanted, HTA in treatment, insulin treated and 
non-insulin treated were considered in the Case Mix group.

\subsubsection{Approach B}

The acquisition of the data used in this approach is made from three different sources: the electronic nursing records, bedside monitors and laboratory. A pre-processing agent is responsible for validating all the collected data and for preparing data to the data mining input table (DMIT). This agent creates the model input structure for a patient, validates the input sources data and processes variables.

For this approach where considered 6 main groups of attributes:

- Case Mix (CM) - Age, admission type, admission from;

- SOFA - Cardiovascular, Respiratory, Renal, Liver, Coagulation and Neurologic;

- ACE (Accumulated Critical Events) - ACE of Blood Pressure (BP), ACE of Oxygen Saturation (SPO2), ACE of Heart Rate (HR) and Total ACE;

- Ratios 1 (R1) - ACE of BP / elapsed time of stay, ACE of SPO2 / elapsed time of stay, ACE of HR / elapsed time of stay, Total of ACE / elapsed time of stay;

- Ratios 2 (R2) - ACE of BP / max number of $\mathrm{ACE}$ of BP, ACE of SPO2 / max number of ACE of SPO2, ACE of HR / max number of ACE of HR;

- Ratios (R) - Union of the two sets of ratios (R1 and R2).

Case Mix consists on a set of variables that are present in the Electronic Health Record (EHR) of the patient. In this approach it was considered the variables age, admission type and admission from. These variables are obtained at patient admission and are automatically transformed according to the DM attributes.

The Sequential Organ Failure Assessment (SOFA) is used in ICU to score the degree of dysfunction/failure of the cardiovascular, respiratory, renal, liver, coagulation and neurological organic systems. The value 0 represents normal function and the maximum value 4 stands for organ failure. In this case the variable used only consider two values $0($ sofa $=0)$ or $1($ sofa $>0)$.

Accumulated Critical Events (ACE) were considered as a complement because SOFA has no predictive capability. ACE include four physiological variables: blood pressure (BP), Oxygen Saturation (SPO2) and Heart Rate (HR).
ACE are calculated by summing the critical event values by hour.

The groups of ratios were added in sequence of the ACE group. These ratios allow determining the number of ACE per hour (R1) and a correspondence between the number of $\mathrm{ACE}$ and the maximum number of events verified in the past, grouped by category and by patient (R2).

Table 2 represents the transformations on the data for this approach as well the variables considered. Note that for Case Mix attributes it is only used one value to represent each case and for the SOFA attributes is considered the worst SOFA value received hourly.

Table 2: Variables used and transformed in approach B.

\begin{tabular}{|c|c|c|c|c|c|}
\hline & ID & Variable & Min & Max & Value \\
\hline & & & 18 & 46 & 1 \\
\hline & $\Delta$ oe & $\Delta$ ar & 47 & 65 & 2 \\
\hline & Mge & Age & 66 & 75 & 3 \\
\hline & & & 76 & 130 & 4 \\
\hline & Admission Tyne & Urgent & - & - & $\mathrm{U}$ \\
\hline & Auminssion 1уре & Programmed & - & - & $\mathrm{P}$ \\
\hline & & Chirurgic & - & - & 1 \\
\hline & & Observation & - & - & 2 \\
\hline & & Emergency & - & - & 3 \\
\hline & Admission From & Nursing & - & - & 4 \\
\hline & & Other ICU & - & - & 5 \\
\hline & & Other & - & - & 6 \\
\hline & & Other & - & - & 7 \\
\hline & Cardiovaccular & $\mathrm{BP}$ (mean) & 0 & 70 & 1 \\
\hline & Caluiovascual & Dopamine & 0.0 & - & 1 \\
\hline & Renal & Dobutamine & 0.0 & - & 1 \\
\hline$\ll$ & Kenal & Epi / Norepi & 0.0 & - & 1 \\
\hline O & Resniratory & Creatinine & 1.2 & - & 1 \\
\hline & & Po2/Fio2 & 0 & 400 & 1 \\
\hline & Hepatic & Bilirubin & 1.2 & - & 1 \\
\hline & Coagulation & Platelets & 0 & 150 & 1 \\
\hline & $\mathrm{ACE}$ & & 0 & $+\infty$ & SET \\
\hline & $\mathrm{R} 1$ & & 0 & 1 & SET \\
\hline & R2 & & 0 & 1 & SET \\
\hline
\end{tabular}

R1, R2 and ACE values were discretised. These values were categorized and grouped considering an interval of values, minimum and maximum. The sets created were defined considering the average and the higher value of data collected. The ranges were created considering the Clinical Global Impression Severity Scale (CGI-S) (Guy, 2000). The criterion used to define percentages concentrate the most part of patient values within a scale between 0 and 5 . Levels 6 and 7 are for severe cases. Table 3 shows the discretization rules.

Table 3: Discretization rules for approach B.

\begin{tabular}{|c|c|c|c|}
\hline \multirow{2}{*}{ Set } & R1 & R2 & \multirow{2}{*}{ Definition } \\
\cline { 2 - 3 } & Average & Maximum & \multirow{2}{*}{} \\
\hline
\end{tabular}




\begin{tabular}{|r|r|r|r|r|l|}
\hline & \multicolumn{1}{|c|}{$>$} & $<=$ & $>$ & $<=$ & \\
\hline 0 & - & $0 \%$ & - & $0 \%$ & Inexistence \\
\hline 1 & $0 \%$ & $25 \%$ & $0 \%$ & $10 \%$ & Normal Condition \\
\hline 2 & $25 \%$ & $50 \%$ & $10 \%$ & $25 \%$ & Borderline Condition \\
\hline 3 & $50 \%$ & $100 \%$ & $25 \%$ & $50 \%$ & Mild Condition \\
\hline 4 & $100 \%$ & $150 \%$ & $50 \%$ & $75 \%$ & Moderate Condition \\
\hline 5 & $150 \%$ & $200 \%$ & $75 \%$ & $90 \%$ & Marked Condition \\
\hline 6 & $200 \%$ & $300 \%$ & $90 \%$ & $100 \%$ & Severe Condition \\
\hline 7 & $300 \%$ & $1000 \%$ & $100 \%$ & $200 \%$ & Extreme Condition \\
\hline
\end{tabular}

Table 4 represents the discretization of the variables. On top there are the different sets and on the left the variables affected.

$\mathrm{R} 1$ classes are determined by the rows $\mathrm{R} 1 \mathrm{BP}$ Min to R1 TOT Max and the R2 attributes (BP, O2, HR and Total) follow the same rule, e.g. for the first level (1) the range is from $0.00(0 \%)$ to $0.10(10 \%)$.

ACE attributes are grouped by their importance and number of occurrences. These values where defined by ICU experts but can be modified in future studies. All these values are calculated in real-time according to the most recent patient results. The values presented in table 4 are the values verified in the moment when the study was performed. These new variables used by the models have as prefix "C_,", e.g. for R2 the class of BP_Max is represented by C_BP_Max.

Table 4: Discretization sets of Data Mining Input.

\begin{tabular}{|c|l|c|c|c|c|c|c|c|r|}
\hline \multicolumn{2}{|c|}{ SET } & \multicolumn{1}{c|}{0} & 1 & 2 & 3 & 4 & 5 & 6 & 7 \\
\hline R1 & Min & -0.100 & 0.000 & 0.010 & 0.021 & 0.041 & 0.062 & 0.082 & 0.123 \\
\cline { 2 - 9 } BP & Max & 0,000 & 0.010 & 0.021 & 0.041 & 0.062 & 0.082 & 0.123 & 2.000 \\
\hline R1 & Min & -0.100 & 0.000 & 0.018 & 0.036 & 0.072 & 0.108 & 0.144 & 0.216 \\
O2 & Max & 0,000 & 0.018 & 0.036 & 0.072 & 0.108 & 0.144 & 0.2162 .000 \\
\hline R1 & Min & -0.100 & 0.000 & 0.004 & 0.008 & 0.015 & 0.023 & 0.0300 .045 \\
\cline { 2 - 9 } HR & Max & 0,000 & 0.004 & 0.008 & 0.015 & 0.023 & 0.030 & 0.0452 .000 \\
\hline R1 & Min & -0.100 & 0.000 & 0.020 & 0.041 & 0.081 & 0.122 & 0.162 & 0.243 \\
TOT & Max & 0.000 & 0.020 & 0.041 & 0.081 & 0.122 & 0.162 & 0.243 & 2.000 \\
\hline \multirow{2}{*}{ R2 } & Min & -0.100 & 0.000 & 0.100 & 0.250 & 0.500 & 0.750 & 0.900 & 1.000 \\
\cline { 2 - 9 } & Max & 0 & 0.100 & 0.250 & 0.50 & 0.750 & 0.900 & 1.0002 .000 \\
\hline \multirow{2}{*}{ ACE } & Min & -0.100 & 0 & 3 & 5 & 8 & 10 & 12 & 15 \\
\cline { 2 - 9 } & Max & 0 & 3 & 5 & 8 & 10 & 12 & 15 & 50 \\
\hline
\end{tabular}

Table 5 presents the distributions of the numeric fields used in approach B. It is possible to observe that the sample data have quite heterogeneous distributions because the variation coefficient is lesser than $20 \%$. This value is used to assess if a distribution is hetero or homogeneous. Table 5 presents the minimum value (MIN), maximum value (MAX), average (AVG), standard deviation (STDDEV) and coefficient of variance (VC).

Table 5: Data Analysis.

\begin{tabular}{|r|r|r|r|r|r|}
\hline Variable & Min & Max & AVG & STDDEV & VC (\%) \\
\hline BP & 0 & 42 & 0.430 & 1.054 & 245.070 \\
\hline
\end{tabular}

\begin{tabular}{|r|r|r|r|r|r|}
\hline BP_Max & 0 & 4.400 & 0.083 & 0.230 & 276.948 \\
\hline BP_Hour & 0 & 0.500 & 0.008 & 0.025 & 331.631 \\
\hline HR & 0 & 16 & 0.500 & 1.474 & 294.800 \\
\hline HR_Max & 0 & 1 & 0.051 & 0.140 & 275.827 \\
\hline HR_Hour & 0 & 0.500 & 0.008 & 0.025 & 305.664 \\
\hline O2 & 0 & 42 & 1.030 & 2.872 & 278.835 \\
\hline O2_Max & 0 & 1 & 0.034 & 0.091 & 267.398 \\
\hline O2_Hour & 0 & 1 & 0.021 & 0.055 & 267.828 \\
\hline Total_ACE & 0 & 50 & 1.970 & 4.340 & 220.305 \\
\hline Total_Max & 0 & 1.170 & 0.052 & 0.108 & 207.934 \\
\hline Total_Hour & 0 & 1 & 0.0370 & 0.077 & 207.751 \\
\hline
\end{tabular}

Table 6 presents the distribution of the classes (in percentage) for each one of the independent variables.

Table 6: Classes Distribution

\begin{tabular}{|r|r|r|}
\hline Variable & Values & Percentage \\
\hline \multirow{2}{*}{ Admission Type } & $\mathrm{P}$ & $23.5 \%$ \\
\cline { 2 - 3 } & $\mathrm{U}$ & $76.5 \%$ \\
\hline \multirow{3}{*}{ Age } & 1 & $16.9 \%$ \\
\cline { 2 - 3 } & 2 & $36.4 \%$ \\
\cline { 2 - 3 } & 3 & $20.8 \%$ \\
\cline { 2 - 3 } & 4 & $25.9 \%$ \\
\hline Respiratory & 1 & $61.6 \%$ \\
\hline Coagulation & 1 & $39.0 \%$ \\
\hline Renal & 1 & $19.7 \%$ \\
\hline Hepatic & 1 & $17.6 \%$ \\
\hline Cardio & 1 & $61.6 \%$ \\
\hline & 1 & $47.3 \%$ \\
\hline \multirow{3}{*}{ Admission From } & 2 & $0.3 \%$ \\
\cline { 2 - 3 } & 3 & $17.7 \%$ \\
\cline { 2 - 3 } & 4 & $14.9 \%$ \\
\cline { 2 - 3 } & 5 & $2.2 \%$ \\
\cline { 2 - 3 } & 6 & $2.0 \%$ \\
\hline & 7 & $15.5 \%$ \\
\hline
\end{tabular}

Figure 3 represents the target (discharge) distribution. The discharged patients are represented by the value 1 , and by 0 are the ones that are hospitalized.

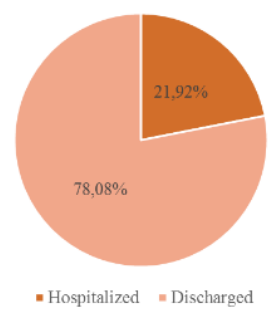

Figure 3: Target Distribution.

\subsection{Modelling}




\subsubsection{Approach A}

In this approach 24 models were created combining 2 different scenarios, 4 target and 3 techniques.

Scenarios:

$$
\begin{aligned}
1= & \{\text { CM, Class EGG_HR, Class SPO2, Class } \\
& \text { SYS, Class DIA, Class Temp }\} \\
2= & \{\text { CM, EGG_HR, SPO2, SYS, DIA, Temp }\}
\end{aligned}
$$
Target:

$$
\begin{aligned}
& 1=\text { Class Hours } 1 \\
& 2=\text { Class Days } 1 \\
& 3=\text { Class Hours } 2 \\
& 4=\text { Class Days } 2
\end{aligned}
$$

Techniques:

$$
\begin{aligned}
& 1=\text { Support Vector Machine } \\
& 2=\text { Decision Trees } \\
& 3=\text { Naïve Bayes }
\end{aligned}
$$

Each one of the targets was defined according to Sturges Rules or with clinical domain provided by ICU physicians.

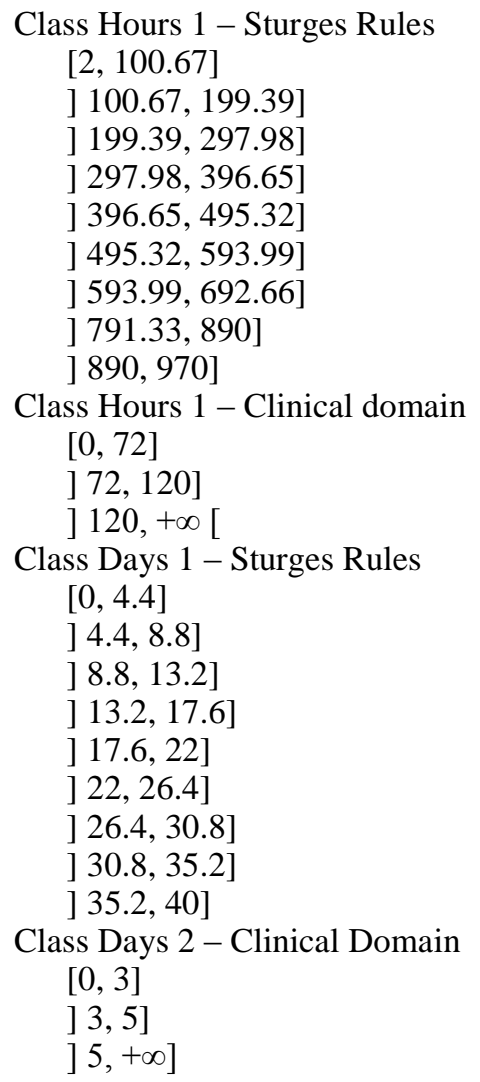

In order to evaluate the models created, $70 \%$ of the data was considered for training and $30 \%$ for test (hold-out sampling). The data used in models corresponds to admissions / discharges made in ICU of CHP from 2011.08.18 to 2014.02.08 (905 days), to 407 patients and to 448 records. The configurations used for these models were based in the ODM pre-defined settings.

\subsubsection{Approach B}

For approach B were developed 39 models. These 39 models combine 13 different scenarios, 1 target and 3 different techniques.

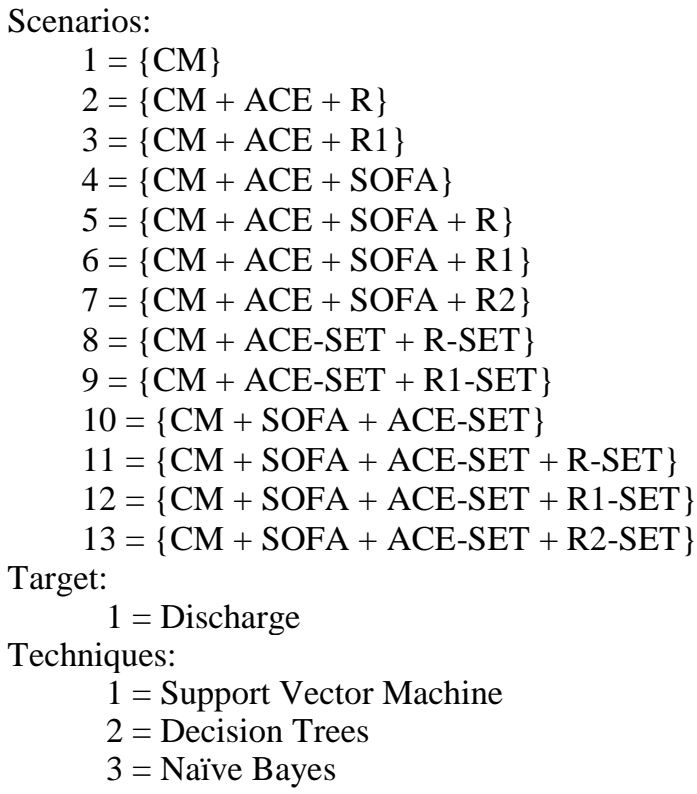

The models created used the same configurations of approach A: $70 \%$ of the data for training and $30 \%$ for testing. The data used in the models correspond to admissions / discharges made in ICU of CHP from 2012.02.01 to 2014.04.24 (813 days), considering 526 patients and 55442 records. Each one of the models has been induced automatically and in real-time using streamed data. The data mining engine used the data present in the input table. This data can be represented as a tuple:

$$
\begin{array}{ccr}
\text { DMIT }=<p i d, & \text { date, hour, } & \text { V_ace_bp, } \\
\text { V_aceBP_time, } & \text { V_aceBP_max, } & \text { V_ace_hr, } \\
\text { V_acehr_time, } & \text { V_aceHR_max, } & \text { V_ace_spo2, } \\
\text { V_ace_spo2_time, } & \text { V_ace_spo2_max, } & \text { V_total_ace, } \\
\text { V_total_ace_time, } & \text { V_total_ace_max, } & \text { V_age, } \\
\text { V_admin_F, } & \text { V_admin_T, } & \text { V_sofa_cardio, } \\
\text { V_sofa_resp, } & \text { V_sofa_renal, } & \text { V_sofa_coag, }
\end{array}
$$$$
\text { V_sofa_hepa> }
$$ 
In this tuple pid represents the patients' identification, the date the date of the values, hour represents the number of hours elapsed since the patient is admitted in the ICU and the tuples with $V$ prefix represent the values obtained for each patient in each date / hour. Table 7 presents the configurations for each one of the algorithms. For each parameter is indicated the values considered and if is a default value or a user-specified value (input).

Table 7: Techniques configurations for approach B.

\begin{tabular}{|r|r|r|l|}
\hline Technique & Setting & \multicolumn{1}{|c|}{ Value } & Type \\
\hline \multirow{4}{*}{ DT } & Minrec Node & 10 & Input \\
\cline { 2 - 4 } & Max Depth & 7 & Input \\
\cline { 2 - 4 } & Minpct Split & 0.1 & Input \\
\cline { 2 - 4 } & Inpurity Metric & Gini & Input \\
\cline { 2 - 4 } & Minrec Split & 20 & Input \\
\cline { 2 - 4 } & Minpct Node & 0.05 & Input \\
\cline { 2 - 4 } NB & Prep Auto & On & Input \\
\hline \multirow{4}{*}{ SVM } & Pairwise Threshold & 0 & Input \\
\cline { 2 - 4 } & Singleton Threshold & 0 & Input \\
\cline { 2 - 4 } & Conv tolerance & 0.001 & Input \\
\cline { 2 - 4 } & Active Learning & Enable & Input \\
\cline { 2 - 4 } & Kernel Function & Linear & Default \\
\cline { 2 - 4 } & Complexity factor & 0.142831 & Default \\
\cline { 2 - 4 } & Prep auto & On & Input \\
\hline
\end{tabular}

\subsection{Results}

\subsubsection{Approach A}

The results obtained by approach A were very poor. The best model presents $73.27 \%$ of overall accuracy. To evaluate these models only was used the accuracy metric, the sensitivity and specificity metrics were not calculated because the outputs are represented by more than two classes. Table 8 presents the three best models for this approach.

Table 8: Three best models for approach A.

\begin{tabular}{|r|r|r|r|}
\hline Scenario & Target & Technique & Accuracy \\
\hline S1 & T2 & NB & $73.28 \%$ \\
\hline S2 & T1 & NB & $73.28 \%$ \\
\hline S2 & T4 & NB & $50.75 \%$ \\
\hline
\end{tabular}

\subsubsection{Approach B}

To evaluate the results obtained by approach B were considered three metrics: sensitivity, accuracy and specificity. As mentioned before sensitivity is the most adequate metric in the medicine area.

Table 9 presents the three best models. Table 10 presents the best models for each metric. This table is useful to find which is the better model when it is expected an equilibrated prediction (accuracy), predict hourly discharge (sensitivity) or predict a non-discharge hour (specificity).

Table 9: Three best models for approach B.

\begin{tabular}{|r|r|r|r|r|}
\hline Scenario & Technique & Accuracy & Sensitivity & Specificity \\
\hline S7 & DT & $74.620 \%$ & $\mathbf{8 7 . 3 2 2 \%}$ & $71.169 \%$ \\
\hline S13 & DT & $77.286 \%$ & $78.355 \%$ & $76.628 \%$ \\
\hline S11 & DT & $77.063 \%$ & $78.673 \%$ & $76.628 \%$ \\
\hline
\end{tabular}

Table 10: Three best models for each metric - approach B.

\begin{tabular}{|c|c|c|}
\hline Accuracy & Sensitivity & \multicolumn{1}{|c|}{ Specificity } \\
\hline S2DT - 77.311\% & S4SVM - 96.140\% & S12DT - 77.067\% \\
\hline S7DT - 77.286\% & S11SVM - 95.963\% & S8DT - 77.043\% \\
\hline S11DT - 77.063\% & S7SVM - 95.902\% & S9DT - 77.043\% \\
\hline
\end{tabular}

To understand the importance of each attribute, a ranking was made for the best model (Table 11). Minimum Description Length, a supervised technique for calculate the attribute importance, was used.

Table 11: Attribute importance for the best model of approach B.

\begin{tabular}{|r|r|r|}
\hline \multicolumn{1}{|c|}{ Attribute } & \multicolumn{1}{|c|}{ Rank } & Importance \\
\hline Hour & 1 & 0.110 \\
\hline EC_AC_TOT & 2 & 0.043 \\
\hline EC_AC_HR & 3 & 0.025 \\
\hline ADMINF & 4 & 0.020 \\
\hline EC_AC_O2 & 5 & 0.019 \\
\hline Age & 6 & 0.010 \\
\hline Cardio & 7 & 0.008 \\
\hline Renal & 8 & 0.002 \\
\hline ADMINT & 9 & 0.001 \\
\hline
\end{tabular}

Considering scenario 7 and the DT technique, the most important attributes correspond to the new variables introduced: number of inpatient hours (Hour), the hourly accumulated critical events (EC_AC) for heart rate and oxygen saturation, the total of accumulated critical events by hour and the place where the patient was previously hospitalized (ADMINF).

\section{DISCUSSION}

The models which presented the best results were the ones that predict the patient discharge for the next hour and were able to update the LOS in real-time. The models of approach A, which predicted the LOS using information of the 
admission process and some measures of patient revealed to be very poor. In the context of medicine these models cannot be considered. DT presented overall accuracies ranging from $7.6 \%$ to $38.46 \%$. For NB the results were situated between $15.14 \%$ and $73.28 \%$. In the case of SVM the worst result corresponds to an accuracy of $32.65 \%$ and the better one to $47.11 \%$. The use of different classes as target does not influence the results (the discretization techniques have no effect in the behaviour of the models). As mentioned before, one of the most important measures for evaluating data mining models in the clinical area is the sensitivity. In the approach A this measure cannot be evaluated because the target variable has more than two classes.

Finalizing, the approach A does not evidence clinical relevance since the corresponding accuracies are very low and does not achieved the baseline defined in accordance with physicians (accuracy > $75 \%$ ). For approach B the models which present best results are the ones that consider case mix, SOFA, $\mathrm{ACE}$ and the second group of ratios (R2). The model that uses Scenario 7 and DT technique presented a good overall accuracy of $74.620 \%$ and a very good sensitivity $87.322 \%$. This model is equilibrated when compared all the metrics. It revels to be a good model to make an accurate prediction of patient discharge. However and being sensitivity one of the most important metrics there are some models which presented sensitivities upper than $95 \%$. In this case the scenario 4 using SVM it is the best model, presenting a sensitivity of $96.140 \%$. In Figure 4 it is possible to observe the ROC for the best model.

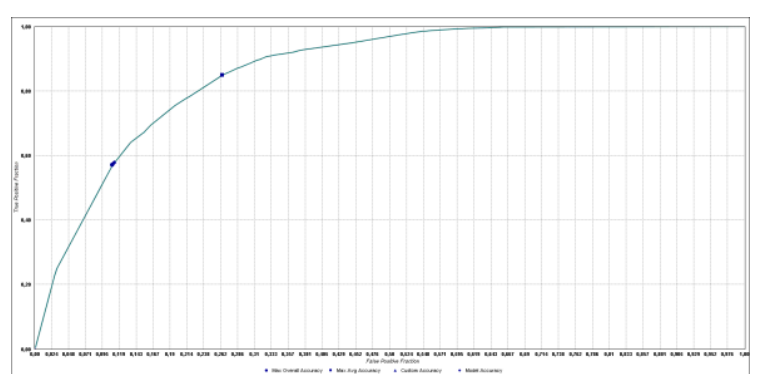

Figure 4: ROC Curve for model S7-DT.

The use of discrete values for ACE and ratios produced models with better accuracies. Nevertheless, sensitivity decreased about $15 \%$ to $20 \%$. Although the efforts made during this work, the accuracy level attained is not very high. The models with better accuracy presented a lower sensitivity. So the choose of the best model depends on the decision-maker objective: good models to predict the possibility of a patient be discharged (sensitivity) or equilibrated models between the two outputs (accuracy). The SVM technique presented very good results in terms of sensitivity but the overall accuracy of these models is very weak. The specificity maintains almost the same results independently the scenario or technique used.

\section{CONCLUSIONS AND FUTURE WORK}

The approaches presented in this work differ in the data set used and in the number classes of the target variable. Approach A considered the worst values occurred during the first 24 hours to predict a number of days (LOS) that ranges from 1 to $n$ ( $n$ classes). Approach B considered real-time values hourly collected to predict if a patient will be discharged in the next hour ( 2 classes). Approach A corresponded to a more conventional approach followed in other areas of medicine. In intensive medicine such approach is not useful. The first part of this work was dedicated to approach A. In the second part, an alternative approach has been explored in order to overcome the limitations encountered in approach A. Approach B revealed characteristics more appropriate to intensive medicine. All the work was carried out under the supervision off ICU physicians. They verified the soundness of the work and the impact of the results in intensive medicine. By the way of this research work interesting results were obtained for predicting LOS in Intensive Medicine. The results allow conclude that the prediction of patient discharge in an hourly base is very efficient. Models induced to predict LOS using admission information and excluding ratios and attributes from the SOFA reveal to be very limited. The discretization of some variables help to increase the overall accuracy of the models however the sensitivity decreases. This approach could not be considered the best due the results presented are not satisfactory.

Intensive Medicine has specific characteristics that make classic LOS models inadequate. Real-time models, able to update the LOS according to the patient condition, obtained better results.

This was an applied research work whose main contributions are:

- Intensive medicine - an efficient predictive model to determine LOS. According to the literature, completely new results were presented; 
- Data mining - a real-time approach has been developed. Results are an interesting increment in the scientific knowledge of streaming data mining.

Those contributions are original and mainly focused in the field of Intensive Medicine. By using Data Mining techniques has been possible achieve a LOS predictive model to improve the discharge decision process. This approach can be followed in other critical units where the patients are in continuous monitoring. In the other cases, solutions presented in the literature can achieve this goal.

In the future additional variables will be considered to understand how they can affect the LOS of inpatients.

\section{ACKNOWLEDGEMENTS}

This work has been supported by FCT Fundação para a Ciência e Tecnologia in the scope of the project: Pest-OE/EEI/UI0319/2014.

The authors would like to thank FCT (Foundation of Science and Technology, Portugal) for the financial support through the contract PTDC/EEI-SII/1302/2012 (INTCare II).

\section{REFERENCES}

Azari, A., Janeja, V.P. \& Mohseni, A., 2012. Predicting Hospital Length of Stay (PHLOS): A Multi-tiered Data Mining Approach. In 2012 IEEE 12th International Conference on Data Mining Workshops. IEEE, pp. 17-24.

Breiman, L. et al., 1984. Classification and Regression Trees no, ed., Belmont, CA: Wadsworth International Group.

Caetano, N. et al., 2014. A Data-Driven Approach to Predict Hospital Length of Stay: A Portuguese Case Study. In ICEIS 2014. (Accepted to be published)

Cios, K. et al., 2007. Supervised Learning: Statistical Methods. In A Knowledge Discovery Approach. Springer US, pp. 307-386.

Clarke, A., 1996. Why are we trying to reduce length of stay? Evaluation of the costs and benefits of reducing time in hospital must start from the objectives that govern change. Quality in health care: QHC, 5(3), pp.172-9.

Dahan, H. et al., 2014. Proactive Data Mining with Decision Trees, New York, NY: Springer New York.

Hachesu, P.R. et al., 2013. Use of data mining techniques to determine and predict length of stay of cardiac patients. Healthcare informatics research, 19(2), pp.121-129.
Han, J., Kamber, M. \& Pei, J., 2011. Data Mining: Concepts and Techniques 3rd ed., San Francisco, CA, USA: Morgan Kaufmann Publishers Inc.

Hand, D.J., Smyth, P. \& Mannila, H., 2001. Principles of data mining. Available at: http://dl.acm.org/citation. $\mathrm{cfm}$ ?id=500820 [Accessed May 5, 2014].

Isken, M.W. \& Rajagopalan, B., 2002. Data Mining to Support Simulation Modeling of Patient Flow in Hospitals. Journal of Medical Systems, 26(2), pp.179197.

Kantardzic, M., 2011. Data Mining: Concepts, Models, Methods, and Algorithms 2nd ed., Wiley-IEEE Press.

Marshall, A., Vasilakis, C. \& El-Darzi, E., 2005. Length of Stay-Based Patient Flow Models: Recent Developments and Future Directions. Health Care Management Science, 8(3), pp.213-220.

Michie, D., Spiegelhalter, D.J., et al., 1994. Machine Learning, Neural and Statistical Classification. Ellis Horwood.

Portela, F., Santos, M.F., Silva, Á., et al., 2013. Data Mining for Real-Time Intelligent Decision Support System in Intensive Care Medicine. In ICAART 2013 - International Conference on Agents and Artificial Intelligence. pp. 270-276.

Portela, F., Santos, M.F., Machado, J., et al., 2013 Pervasive and Intelligent Decision Support in Critical Health Care Using Ensembles. In ITBAM. pp. 1-16.

Portela, F., Pinto, F. \& Santos, M.F., 2012. Data Mining Predictive Models for Pervasive Intelligent Decision Support in Intensive Care Medicine. In KMIS. pp. 8188.

Quiland, J.R., 1986. Induction of Decision Tress. Machine Learning, 1(1), pp.81.106.

Rokach, L. \& Maimon, O., 2010. Supervised Learning. In O. Maimon \& L. Rokach, eds. Data Mining and Knowledge Discovery Handbook. Springer US, pp. 133-147.

Santos, M.F. \& Azevedo, C., 2005. Data Mining Descoberta de conhecimento em base de dados, FCA Editora de Informática, Lda.

Santos, M.F., Portela, F. \& Vilas-Boas, M., 2011. INTCARE: multi-agent approach for real-time intelligent decision support in intensive medicine. Taylor, K., 2010. Oracle Data Mining Concepts.

Vapnik, V.N., 1995. The Nature of Statistical Learning Theory 1st ed., Springer.

Wagener, G. et al., 2011. The Surgical Procedure Assessment (SPA) score predicts intensive care unit length of stay after cardiac surgery. The Journal of thoracic and cardiovascular surgery, 142(2), pp.44350.

Zhang, X.-C., Zhang, Z.-D. \& Huang, D.-S., 2012. Prediction of length of ICU stay using data-mining techniques: an example of old critically Ill postoperative gastric cancer patients. Asian Pacific journal of cancer prevention: APJCP, 13(1), pp.97101 